\title{
The prospects of layered alumino-silicates in the synthesis of nano-excipients for controlled release systems
}

\begin{abstract}
Over the past six decades, there has been a growing need for designing controlled release formulations of drugs and agrochemicals in order to attain an extended therapeutic effect of various active ingredients, improve patient convenience, maintain steady state of active ingredients-target site interaction, optimize release kinetics, reduce side effects as well as improve the way in which the active ingredients are delivered into the targeted organ. On account of that, researchers in the course of time have developed three generations of controlled release systems (CRSs) namely, first generation which consist of basics of controlled release systems; second generation which consist of smart delivery systems; and third generation which consist of modulated delivery systems. In view of challenges emanating from CRS usage such as difficulties in retrieving active ingredients upon hypersensitivity, decreased systemic availability as well as poor in vitro-in vivo correlation, the need to further the design of CRSs using nano-excipients set-in. Consequently, opportunities aiming at synthesizing effective nano-based-excipients of active ingredients using in situ polymerization, graft polymerization, co- polymerization and intercalation emerged. With regard to intercalation technique, application of 2:1 layered nano materials as nano-excipients has been a success leaving behind a question of what limits the application of 1:1 layered nano materials as nano-excipients in CRS industry. This paper therefore, intends to provide an overview of progress made in the application of 1:1 layered nanomaterials as nano-excipients by using first generation intercalation compounds technically called intermediate intercalation agents.
\end{abstract}

Keywords: Controlled release systems, Excipients, Layered clays, Drug delivery systems, Controlled release fertilizers, Intercalations, Nanotechnology
Volume 5 Issue 5 - 2017

\section{Sempeho Ibahati Siafu}

Department of Distance Education, Institute of Adult Education, Tanzania

Correspondence: Sempeho Ibahati Siafu, Department of Distance Education, Institute of Adult Education, P.O. Box 20679 Dar es Salaam,Tanzania, Email sempeho@iae.ac.tz

Received: April 28, 2017| Published: June 06, 2017

\section{Introduction}

As often as not, controlled release systems (CRSs) have been a topic of interest for researchers over the past 6 decades $^{1}$ due the inherent properties associated with the controlled release formulation. As a matter of fact, ${ }^{2}$ demonstrated that "CRSs have been devised to enable superior control of drug exposure over time, to assist drug in crossing physiological barriers, to shield drug from premature elimination, and to shepherd drug to the desired site of action while minimizing drug exposure elsewhere in the body". Similarly, it has been established that, due to enhanced performance of controlled release formulations relative to their conventional counterparts, CRS reduce the frequency of administration of active ingredients which in our context include drugs [under drug delivery systems (DDSs)] and agrochemical [under controlled release fertilizers (CRFs)]. ${ }^{3}$

As far as nanotechnology is concerned, the design of CRS is achieved by using nano-excipients or literally nano-carriers. At times where a synergy between nano-excipients is needed to further improve the performance of CRSs, nanocomposite excipients may be used. What is more, when polymeric excipients are used alone as excipients the word nano-excipient may be used if the diameter of resulting particles is below $100 \mathrm{~nm}$. Examples of nano-excipients include among others, microemulsions, liposomes, dendrimers, block polymer micelles, solid lipid and polymer nanoparticles along with soluble polymers with drug attached on the side chains by biodegradable linkages. ${ }^{2}$ On the other hand, when the interest is to use intercalation compound utilizing layered aluminosilicates along with simultaneous encapsulation of the intercalation compound with polymeric materials the composite excipient results; if the composite excipients are again possessing particle diameter of below $100 \mathrm{~nm}$ the term nano-composite excipients hold true. Precisely, this may involve inserting drug molecules into the interlayer spaces of nanolayers of aluminosilicates followed by encapsulation with biodegradable polymers. ${ }^{4,5}$

In times gone by, the progression of CRS can be traces by considering three generations of CRS researches namely, first generation which consist of basics of controlled release systems; second generation which consist of smart delivery systems; and third generation which consist of modulated delivery systems as seen in Table 1. The details of the information presented in the Table 1 can be found elsewhere. ${ }^{1}$

Table I Evolution of controlled release systems over the past 6 decades

\begin{tabular}{|c|c|c|}
\hline $\begin{array}{l}\text { It Generation } \\
\text { (1950's to 1980) }\end{array}$ & $\begin{array}{l}2^{\text {nd }} \text { Generation } \\
(1980 \text { to } 2010)\end{array}$ & $\begin{array}{l}3^{\text {rd }} \text { Generation (20 I 0- } \\
2040)\end{array}$ \\
\hline $\begin{array}{l}\text { Basics of } \\
\text { controlled release }\end{array}$ & $\begin{array}{l}\text { Smart delivery } \\
\text { systems }\end{array}$ & $\begin{array}{l}\text { Modulated delivery } \\
\text { systems }\end{array}$ \\
\hline Oral delivery & Zero order release & On-off insulin release \\
\hline Transdermal delivery & $\begin{array}{l}\text { Smart polymers \& } \\
\text { hydrogels }\end{array}$ & Targeted delivery \\
\hline \multirow[t]{2}{*}{$\begin{array}{l}\text { Drug release } \\
\text { mechanisms }\end{array}$} & $\begin{array}{l}\text { Peptide \& protein } \\
\text { delivery }\end{array}$ & $\begin{array}{l}\text { Long term delivery } \\
\text { systems }\end{array}$ \\
\hline & Nanoparticles & In vitro-in vivo correlation \\
\hline
\end{tabular}

Meanwhile, the evolution of controlled dosage forms relative to conventional or immediate release dosage forms is well established..$^{1,6}$ As a matter of fact, literature states that "many of the present-day approaches for achieving controlled release from coated forms 
are not new other than the involvement of newer coating materials and excipients...excipients known at the time include wax, shellac, nitrated cellulose lacquers, or gum benzoin as coating material; agar, cornmeal, or kaolin as hygroscopic swelling agent; mixture of tartaric acid and sodium bicarbonate as effervescent agent; along with sodium chloride or sugar soluble additive" etc ${ }^{7}$ Despite of all the shortcoming of the CRSs, ${ }^{8}$ literature clearly shows outstanding contribution of CRSs in the current dynamic world. ${ }^{9-15}$ In other words, trends indicates overwhelming needs for research regarding CRSs owing to the fact that the strengths of formulations emanating from the application CRSs outnumbers the weaknesses. ${ }^{16-20}$ Besides, CRSs trends reveal an integrated approach towards the design of the excipients materials from polymeric excipients. Layered aluminosilicates as well as a synergy between them along with an advanced approach which capitalizes on the application of nanotechnology to design nanocomposites excipients. . $^{21,23-25}$ It is the purpose of this paper to present a summarized detailed of what has transpired in the application of polymeric and layered silicates in the CRS industry. In particular, the discussion will be on the applications of layered aluminosilicates such as smectites and kaolinite for preparation of nanocomposites excipients utilizing intercalation reaction. This is due to known potential opportunities for research $^{26-29}$ regarding CRS using layered silicates.

\section{Approaches to controlled release systems}

Essentially, CRSs have been synthesized by using a number of synthetic pathways; hereto is a summary of some of these pathways:

\section{In-situ polymerization}

This involves loading of the active ingredients into the reaction mixture followed by in-situ polymerization; ${ }^{30}$ in so doing the active ingredient get entrapped within the polymeric matrix thereby generating an active ingredient-polymeric complex. In such cases, the polymeric matrix acts as a career molecule functioning as an excipient of the named active ingredient. The challenge behind application of this approach in the design of excipients molecules is that: the active ingredient may influence the polymerization process and the resulting polymer network structure. It is necessary to note that, when the interaction between active ingredient and the polymeric excipient follows physisorption reaction the synthesis is successful due to likelihood that upon swelling of the excipients (as a result of some mechanisms described in $^{31}$ the active ingredient can be detached and get released into the target organ. This is opposed to the chemisorptions interactions in which the interaction between the active ingredient and the excipients proceed into a chemical change and the result is not reversible. As a matter of fact a release phenomenon is a function of physisorption interaction between excipients and the active ingredients.

\section{Graft polymerization}

In this experimental approach, the dry polymeric material is allowed to swell in the solution containing the active ingredient and "after equilibrium swelling the polymeric excipient is dried"; this result in the formation of a complex containing active ingredient encapsulated into the polymeric excipient. ${ }^{31-33}$ Contrary to the in-situ polymerization described before, this approach may be associated with the accumulation of the loaded active ingredient on the surface during the drying of the loaded hydrogel and as a result bursting effect may set-in. Basically, researches regarding the design of nanocomposite excipient are limited by the occurrence of bursting effect in the synthesized CRS formulations. As a matter of fact, opportunities for further researches are massive in this area wherein, attempts towards minimizing bursting effect of the CRS formulation upon ingestion are virtually needed. On top of that, this approach is also associated with low loading amount particularly when the active ingredient affects the water absorbency characteristics of synthesized CRS formulations. Concretely, the preparation of CRS formulations by way of using nanocomposite excipients may be enhanced by the application of some physical methods. According to Ukessays, ${ }^{34}$ some of the physical methods which have been employed so far in the encapsulation of active ingredients into the excipients materials include but not limited to spray coating, spray drying, pan coating, rotary disk atomization, rotary drum, pan mix, ribbon mix, paddle mix and fluidized bed. Besides, manual reflux systems have also being used in the course of CRS synthesis.

\section{Intercalation reactions}

These are merely topochemical reactions which involves the insertion of an active ingredient into a "host lattice whereby the guesthost structure or intercalation compound is only slightly perturbed from the host structure and that, the reaction used to form the compound is reversible. ${ }^{4,35}$ Layered aluminosilicates are made of high aspect ratio nanolayers. The suitability of layered amuninosilicate nanolayers ${ }^{36-39}$ as excipient molecules for design of different CRS formulations is based on the following factors:

Surface area and reactivity: Layered aluminosilicates nanolayers posses large surface area and reactivity that is greater than a micrometer sizes materials and hence suited for use as a career molecule

Nature of Surfaces and Interfaces: The surfaces and interfaces of nanolayers contained in the have functional groups whose chemical properties serve as active substrates for physical, chemical, biological reactions. Actually, possession of these properties makes aluminosilicates ever better suited in the synthesis of anti-acids, cosmetics etc.

Presence of Interlayer Spaces: Fundamentally, amuninosilicates may be classified as 2:1 or 1:1 layered. Smectites are a good example of 2:1 while kaolins are examples of 1:1 layered silicates. Structurally, 2:1 amuninosilicates (Figure 1) are formed when one alumina octahedral sheet is sandwiched between two silica tetrahedral sheets as in seen in Figure 3 of. ${ }^{31}$ On the other hand, 1:1 layered amuninosilicates are formed when one alumina octahedral sheet is sandwiched with one silica tetrahedral sheet (Figure 2); the details of structural arrangements used here can be found in..$^{40}$ As seen in Figure $1 \& 2$, there seems an appearance of interlayer space between T-T sheets for 2:1 layered and O-T sheets for 1:1 aluminosilicates. According to, ${ }^{41}$ the interlayer spaces contain positive cations and water molecules as seen in Figure 3 of. ${ }^{31}$ In other words, the interlayer spaces contain exchangeable cations necessary for the compensation of negative charges developed during ion-substitution taking place at the sheets. Basically, site vacancies or ion substitution reaction makes it possible for layered aluminosilicates to be used as excipients molecules. Most reactions involve the substitution of exchangeable ions with active ingredients. Notably, the application of layered silicates has been favoured by researchers since the interactions resulting from substitution are merely physical or rather physisorption.

Other properties: According to Joshi $\mathrm{GV},{ }^{42}$ most naturally available layered aluminosilicates are also associated with some properties that makes them suitable for use as excipients molecules. Such properties include, adsorptive capacity, rheological properties, chemical inertness and low or null toxicity. It may be noted that, excipients do as well interact with tissues of organism through which the CRS formulation are made for. Therefore, it is necessary that excipient materials be 
inert with null toxicity otherwise, critical side effects and other fatal conditions may happen once the synthesized CRS formulations are ingested.
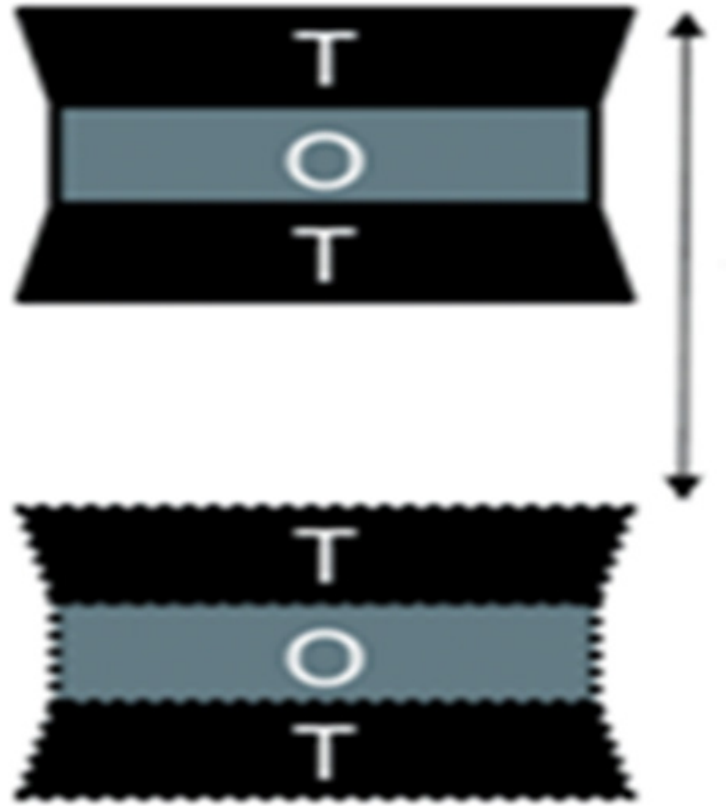

Figure I A 2:I configuration of layered aluminosilicates. ${ }^{51}$

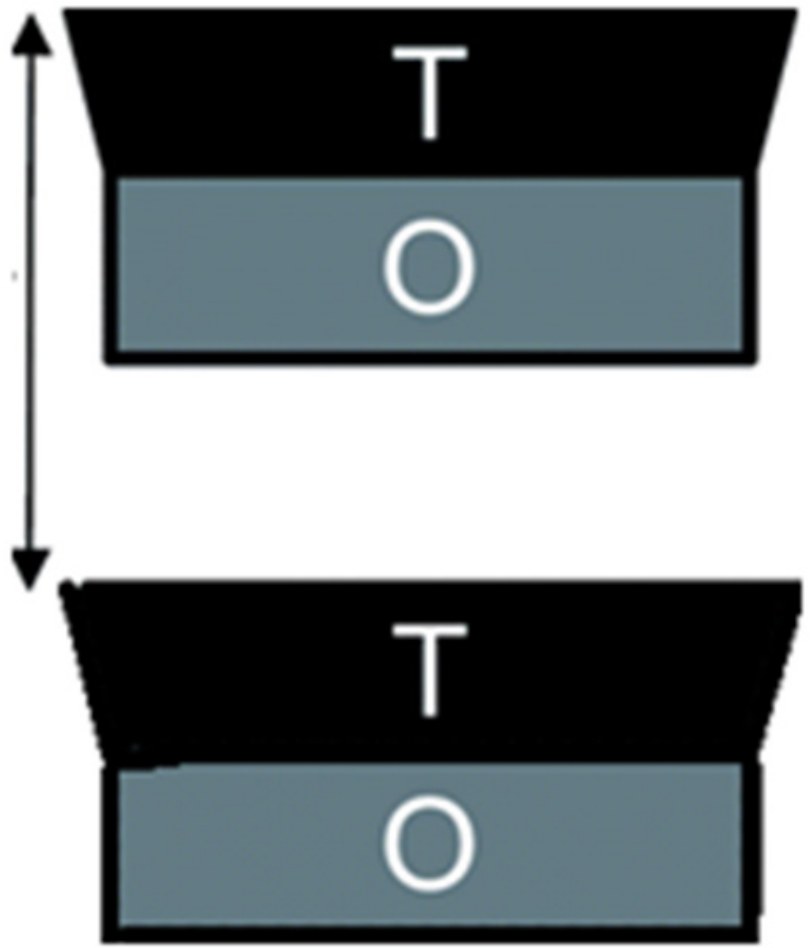

Figure $2 \mathrm{~A}$ I: I configuration of layered aluminosilicates..$^{51}$

\section{Application of 2:I Layered Alumino-silicates in the Synthesis of Nanocomposite Excipients}

Hitherto, there are a good number of studies that have been carried out to investigate the application of 2:1 layered aluminosilicates in the synthesis of nanocomposite excipients. Hybrids of montmorillonite
(MTT) and various active ingredients have received much attention by researchers owing to the aforementioned properties of aluminosilicates. Actually, a good example is the study conducted by Flávia Carmo Horta Pinto ${ }^{43}$ in which MTT was used as an excipient for vitamin B1. An ion exchange reaction at the interlayer spaces of MTT was a driving force behind the reaction mechanism. The results for that study indicated that MTT is a suitable excipient for design of vitamin B1 CRS formulations and that the release of the vitamin B1 as an active ingredient followed Higuchi kinetic model and the diffusion-controlled mechanism and it was a function of $\mathrm{pH}$. The details of CRSs models can be found elsewhere. ${ }^{35}$ Similarly, a study by Ambrogi $\mathrm{V}^{44}$ has indicated the suitability of MTT as an excipient of Triamcinolone Acetonide (TA). As a matter of fact, the study as demonstrated that MTT can be used to prepare implantable drug delivery systems (DDSs) (a form of CRSs) capable of being able to control the release of a therapeutic agent to a specific target to treat or prevent different types of pathological processes.

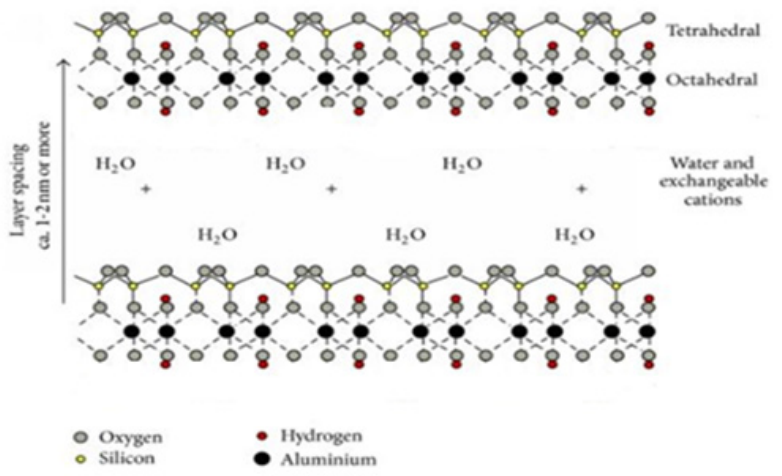

Figure 3 Kaolinite I:I molecular structure arrangement.

Conspicuously, lots more studies about the application of 2:1 layered aluminosilicates in the synthesis of nanocomposites are currently known in literature owing to the nature of T-O-T symmetric arrangement of sheets as seen in Figure 1.44-46 The T-O-T symmetric arrangement of sheets in 2:1 layered aluminosilicates ensures that "cation exchangeable region interlayer spaces can accept the introduced species by way of isomorphous substitution reaction and that process results in the expansion of the interlayer distance between the kaolinite layers". ${ }^{47-52}$

Furthermore, most researches involving the 2:1 layered aluminosilicates in the synthesis of nanocomposites proceed with a simple methodological procedures involving sample purification especially with $\mathrm{NaCl}$ solution following by analysis with a number of techniques. Solid dispersion technique has been commonly used in these studies wherein, shaking of solutions of active ingredients and excipient materials are done. Optimization of $\mathrm{pH}$, temperature and concentrations along with release profiles have been issues of interest for most investigations. ${ }^{41}$ In most cases the parameters of interest in these studies have been the influences of $\mathrm{pH}$, adsorption isotherms, thermodynamic properties, morphological changes, particles size and their impacts as well as release kinetics following both in vivo and in vitro release phenomena. ${ }^{34,41,53}$

\section{Limitations of Using I:I Layered Alumino- silicates in the Synthesis of Nanocomposite Excipients}

Thus far, literature has hypothesized that for the most cases, 1:1 layered aluminosilicates are reluctant to most reactions which 
involves insertion of guest molecules such as active ingredients, biological species etc., into the interlayer spaces. Taking kaolinite as a case study, this hypothetical standpoint can be put clear by looking into the 1:1 molecular structure of kaolinite (Figure 3). Considering 2:1 structure which is T-O-T:T-O-T (Figure 1), the configuration present symmetrical arrangement of $\mathrm{T}-\mathrm{T}$ interfaces; this is opposed to 1:1 structure which is T-O:T-O as seen in Figure $2 \& 3$ wherein the configuration present asymmetrical arrangement of O-T interfaces. Succinctly, the differences between 2:1 and 1:1 layered aluminosilicates is also based on their configurations which are symmetrical and asymmetrical configurations for the 2:1 and 1:1 layered aluminosilicates respectively. Actually, according to $\mathrm{Bi}$ $\mathrm{X},{ }^{39}$ asymmetrical configuration is associated with little substitution in structural lattices thereby giving rise to low exchange capacity $(\approx 3-15$ meq per $100 \mathrm{~g}$ for kaolinite $)$ due to a minimal layer charge; consequently, low tendency for ion substitution which is critical for most intercalation reactions occur. ${ }^{10,54,55}$ This phenomenon can be attributed to the fact that, asymmetric structure in 1:1 layered aluminosilicates allows the formation of hydrogen bonds between consecutive layers, providing a large cohesive energy. In turn, this makes intercalation of active ingredients more difficult. ${ }^{39}$ In fact, this may probably be the main reason that has in the course of time limited the application 1:1 layered aluminosilicates in the synthesis of nanocomposite excipients.

\section{Utilization of Intermediate Intercalation Compounds in the Synthesis of $1: 1$ Based Nanocomposite Excipients}

Outstandingly, due to the aforementioned limitations of using 1:1 layered aluminosilicates in the synthesis of nanocomposite excipients, scientists in the course of time have endeavored to investigate this phenomena in attempt to find out how the electrostatic and chemical forces emanating from asymmetric configuration can be overcome thereby unveiling the prospects of using 1:1 layered aluminosilicates in the reactions involving insertion of active ingredients into the interlayer spaces. In the first place, researchers had found that just sheer adsorption phenomenon govern the chemistry of the aqueous reaction involving layered aluminosilicates containing 1:1 configuration. ${ }^{35,56}$ In point of fact, it was observed that when active ingredients are intercalated into the 1:1 interlayer spaces, the outcome is the adsorption of the active ingredients on the lower and upper surfaces of the functional sheets instead of being intercalated within the interlayer spaces. Even so, further studies revealed that 1:1 interlayer spaces can be expanded to allow intercalation reactions to proceed; this was possible by applying macromolecular complexes capable of being able to overcome the limiting electrostatic and chemical forces. These macromolecular complexes I have named as first generation guest molecules or in more technical term, I have named them as "intermediate intercalation compounds". Characteristically, after reading several literature, the criteria which were examined and found to be used by to judge whether a particular macromolecular complex is fitting as intermediate intercalation compounds include: (i) the ability to overcome the limiting electrostatic and chemical forces without causing chemical change into the interlayer spaces, (ii) the ability to undergo isomorphous substitution with the second generation intercalation compound or rather the incoming active ingredient and, (iii) inertness or inability to modify the nature and chemical properties of the active ingredient during substitution process. ${ }^{57}$

Owing to that, studies have been carried out to investigate the suitability of various natural and synthetic macromolecules for use as the intermediate intercalation compounds and name a few include,
DMSO, methanol, chlorhydrol etc. Therefore, investigations regarding the likelihood of occurrence of interactions between intermediate intercalation compounds with the active ingredients which may limit their reactivity upon reaching the target organs are called for. Similarly, the question of the mechanism which governs isomorphous substitution between first generation intercalation compounds (i.e. intermediate intercalation compounds) and the second generation intercalation compound (active ingredients) is yet to be exhausted though attempts are on the go. Also, the issue pertaining to the nature of electrostatic and chemical forces operating in 2:1 vis-à-vis 1:1 configurations of layered aluminosilicates is still irony. Such question stands as opportunities for contemporary researches regarding the application of layered silicates in the preparation of advanced nanocomposite excipients.

\section{Conclusion}

Advances in the studies related to the interaction between active ingredients and carrier molecules or delivery devices have brought technological transformations in the chemical conventional formulations (CCFs) of active ingredients to the controlled release formulations (CRSs) such drug delivery systems (DDS) and controlled release of agrochemicals (CRFs). Transition from CCFs to CRSs was a function of studies which led to the emergence of active ingredients' carriers or excipients. Application of excipient in the design of CRSs has revolutionalized the world of chemical investigations such that, researches are currently directed towards designing CRSs using nanoexcipients as well as nanocomposite excipients. As yet, the subject of CRSs particularly in the application of polymerizable materials, natural hydrogels as well as layered silicates containing functional nano-layers has opened up copious prospects for researchers as described earlier in this paper. Besides, nano-technological advances have led to the development of design of CRSs by way of applying nanocomposite excipients. Nanocomposite excipients have been demonstrated to offer opportunities to study the effect of the synergy between excipients materials/molecules in the nano-meter scale. Research has demonstrated improved properties of most CRSs which bring into play nanocomposite excipients as delivery devices of active ingredients. In so speaking, it is equally important for researchers to opt for applying nanocomposite excipients especially those which involve a amalgamation of layered aluminosilicates which uses intercalation as well as hydrogels which employ polymerization or encapsulation in order to study the influence of synergy offered by nanocomposite excipients in the design of CRSs.

\section{Acknowledgments}

None.

\section{Conflict of interest}

None.

\section{References}

1. Park K. Controlled drug delivery systems: past forward and future back. J Control Release. 2014;190:3-8.

2. Siegel RA, MJ Rathbone. Overview of Controlled Release Mechanisms. Fundamentals and Applications of Controlled Release Drug Delivery. Controlled Release Society, Advances in Delivery Science and Technology. 2012;p:19-43.

3. Shaviv A. Advances in controlled-release fertilizers. Advances in agronomy. 2001;71:1-49.

4. Jacobson AJ, LF Nazar. Intercalation Chemistry: Encyclopedia of Inorganic Chemistry, JohnWiley \& Sons, USA. 2006. 
5. Zuckerman JJ, AP Hagen. Inorganic Reactions and Methods, Oligomerization and Polymerization Formation of Intercalation Compounds, VCH Publishers, Wiley, USA. 2009.

6. Friend DR. Development of controlled release systems over the past 50years in the area of contraception. Journal of Controlled Release. 2016;240:235-241.

7. Lee PI, J Li. Evolution of oral dosage controlled release dosage forms. John Wiley \& Sons, Inc, USA. 2010.

8. Dikmen G, Genc L. Advantages and Disadvantages in Drug Delivery Systems. Journal of Materials Science and Engineering. 5(2011):468-472.

9. Thompson HO, Lee CO. History, and theory of enteric coatings. $J$ Am Pharm Assoc. 1945;34(5):135-138.

10. Schneider $H$, Nightingal C. Evaluation of an oral prolongedrelease antibiotic formulation. Journal of pharmaceutical sciences. 1978;67(11):1620-1622.

11. Senel S, Ikinci G, Kaş S, et al. Chitosan films and hydrogels of chlorhexidine gluconate for oral mucosal delivery. Int $J$ Pharm. 2000;193(2):197-203.

12. Pakulska MM, S Miersch, Molly S Shoichet. Designer protein delivery: From natural to engineered affinity-controlled release systems. Science. 2016;351(6279):aac4750

13. Guerin J, J Burgain, Frédéric Borges, et al. Use of imaging techniques to identify efficient controlled release systems of Lactobacillus rhamnosus GG during in vitro digestion. Food \& Function. 2017;p.4.

14. Lin YJ, Huang CC, Wan WL, et al. Recent Advances in $\mathrm{CO}_{2}$ BubbleGenerating Carrier Systems for Localized Controlled Release. Biomaterials. 2017;133:154-164.

15. Yegin, Y, G Yilmaz, Ömer Karakoç, et al. Molecularly Imprinted Materials for Controlled Release Systems. Advanced Molecularly Imprinting Materials. 2017;pp.455-522.

16. Robinson JR. Sustained and controlled release drug delivery systems, M. Dekker. 1978

17. Sastry SV, Nyshadham JR, Fix JA, et al. Recent technological advances in oral drug delivery-a review. Pharmaceutical science \& technology today. 2000;3(4):138-145.

18. Pillai O, R Panchagnula. Polymers in drug delivery. Curr Opin Chem Biol. 2001;5(4):447-451.

19. Gupta P, K Vermani, Garg S. Hydrogels: from controlled release to $\mathrm{pH}-$ responsive drug delivery. Drug Discov Today. 2002;7(10):569-579.

20. Patri AK, Majoros IJ, Baker JR. Dendritic polymer macromolecular carriers for drug delivery. Curr Opin Chem Biol. 2002;6(4):466-471.

21. Barahuie Farahnaz, Hussein Mohd Zobir, Arulselvan Palanisamy, et al. Development of the anticancer potential of a chlorogenate-zinc layered hydroxide nanohybrid with controlled release property against various cancer cells. Science of Advanced Materials. 2013;5(12):1983-1993.

22. Barahuie F, MZ Hussein. Development of drug delivery systems based on layered hydroxides for nanomedicine. International journal of molecular sciences. 2014;15(5):7750-7786.

23. Zhang K, ZP Xu, Ji Lu, et al. Potential for layered double hydroxidesbased, innovative drug delivery systems. International journal of molecular sciences. 2014;15(5):7409-7428.

24. Pang X, J Cheng, Li Chen, et al. The preparation and characterization of lactone form of 10-hydroxycamptothecin-layered double hydroxide nanohybrids. Applied Clay Science. 2015;104:128-134.

25. Barahuie F, Hussein MZ, Arulselvan $\mathrm{P}$, et al. Controlled in vitro release of the anticancer drug chlorogenic acid using magnesium/aluminiumlayered double hydroxide as a nanomatrix. Science of Advanced Materials. 2016;8(3):501-513.
26. Aguzzi CP, Cerezo. Use of clays as drug delivery systems: possibilities and limitations. Applied Clay Science. 2007;36(1):22-36.

27. Suresh R, Borkar S, Sawant VA, et al. Nanoclay drug delivery system. International Journal of Pharmaceutical Sciences and Nanotechnology. 2010;3(2):901-905

28. Viseras C, P Cerezo. Current challenges in clay minerals for drug delivery. Applied Clay Science. 2010;48(3):291-295.

29. Seema, Datta Mounika. MMT-PLGA nanocomposites as an oral and controlled release carrier for 5-fluorouracil: a novel approach. Int $J$ Pharm Pharm Sci. 2013;5(2):332-341.

30. Jordan J, KI Jacob, Rina Tannenbaum, Mohammed A Sharaf, et al. Experimental trends in polymer nanocomposites-a review. Materials science and engineering: A. 2005;393(1):1-11.

31. Sempeho, Hee Taik Kim, Egid Mubofu, and Askwar Hilonga. Meticulous Overview on the Controlled Release Fertilizers. Advances in Chemistry. 2014:16.

32. Bhattacharya A, B Misra. Grafting: a versatile means to modify polymers: techniques, factors and applications. Progress in polymer science. 2004;29(8):767-814.

33. Levina M, Rajabi-Siahboomi AR. The influence of excipients on drug release from hydroxypropyl methylcellulose matrices. J Pharm Sci. 2004;93(11):2746-2754.

34. Ukessays. Controlled Release Fertilizers and Nanotechnology Traces. Biology Essay. 2013.

35. Grillo R, Pereira Ado E, De Melo NF, et al. Controlled release system for ametryn using polymer microspheres: preparation, characterization and release kinetics in water. J Hazard Mater. 2011;186(2):1645-1651.

36. Siafu Ibahati Sempeho, Hee Taik Kim, Egid Mubofu, et al. Dynamics of Kaolinite-Urea Nanocomposites via Coupled DMSOHydroxyaluminum Oligomeric Intermediates. Indian Journal of Materials Science. 2015:1-10.

37. Park MC, Kim H, Park DH, et al. Ketoprofen-LDH nanohybrid for transdermal drug delivery system. $B$ Kor Chem Soc. 2012;33(6):1827-1828.

38. Abdeen R, N Salahuddin. Modified chitosan-clay nanocomposite as a drug delivery system intercalation and in vitro release of ibuprofen. Journal of Chemistry. 2013:9.

39. Bi X, H Zhang, Liguang Dou. Layered double hydroxide-based nanocarriers for drug delivery. Pharmaceutics. 2014;6(2):298-332.

40. Unalan IU, G Cerri. Nanocomposite films and coatings using inorganic nanobuilding blocks (NBB): current applications and future opportunities in the food packaging sector. RSC advances. 2014;4(56):29393-29428.

41. Parolo M, Fernández L, Zajonkovsky I, et al. Antibacterial activity of materials synthesized from clay minerals. Science against microbial pathogens: communicating current research and technological advances. Formatex, Microbiology series. 2011;3:144-151.

42. Joshi GV, HA Patel. Montmorillonite intercalated with vitamin B1 as drug carrier. Applied Clay Science. 2009;45(2009):248-253.

43. Flávia Carmo Horta Pinto, Armando Silva-Cunha, Gerson Antônio Pianetti, et al. Montmorillonite Clay-Based Polyurethane Nanocomposite As Local Triamcinolone Acetonide Delivery System. Journal of Nanomaterials. 2011:1-11.

44. Ambrogi V, Fardella G, Grandolini G, et al. Intercalation compounds of hydrotalcite-like anionic clays with antiinflammatory agents-I Intercalation and in vitro release of ibuprofen. Int J Pharm. 2001;220(1):23-32.

45. Choy JH, SJ Choi, Taeun Park, et al. Clay minerals and layered double hydroxides for novel biological applications. Applied Clay Science. 2007;36(1):122-132. 
46. Kuthati Y, Kankala RK, Chia Hung Lee. Layered double hydroxide nanoparticles for biomedical applications: Current status and recent prospects. Applied Clay Science. 2015;112:100-116.

47. Laszlo P. Chemical reactions on clays. Science. 1987;235(4795):1473-1478

48. Vaia RA, H Ishii, Emmanuel P Giannelis. Synthesis and properties of two-dimensional nanostructures by direct intercalation of polymer melts in layered silicates. Chemistry of Materials. 1993;5(12):1694-1696.

49. Alexandre M, Dubois P. Polymer-layered silicate nanocomposites: preparation, properties and uses of a new class of materials. Materials Science and Engineering: R: Reports. 2000;28(1-2):1-63.

50. Davis Ss. The design and evaluation of controlled release systems for the gastrointestinal tract. Journal of Controlled Release. 1985;2:27-38.

51. Kornmann X, Lindberg H, Berglund LA. Synthesis of epoxy-clay nanocomposites: influence of the nature of the clay on structure. Polymer. 2001;42(10):1303-1310.
52. Rajamathi M, GS Thomas, P Vishnu Kamath. The many ways of making anionic clays. Journal of Chemical Sciences. 2001;113(5-6):671-680.

53. Park JK, Choy YB, Oh JM, et al. Controlled release of donepezil intercalated in smectite clays. Int J Pharm. 2008;359(1):198-204.

54. Vaughan D. Pillared clays-a historical perspective. Catalysis Today 1988;2(2):187-198.

55. Bergaya F, G Lagaly. General introduction: clays, clay minerals, and clay science. Developments in clay science. 2006;1:1-18.

56. Siafu Ibahati Sempeho, Hee Taik Kim, Egid Mubofu, et al. Encapsulated Urea-Kaolinite Nanocomposite for Controlled Release Fertilizer Formulations. Journal of Chemistry. 2015:17.

57. Siafu ISS. Studies on the Nanocomposite Excipients for Controlled Release Fertilizers. Materials Sciences and Engineering. Nelson Mandela African Institution of Science and Technology, Tanzania. 2016. 